Research Paper

\title{
Doxycycline Inducible Melanogenic Vaccinia Virus as Theranostic Anti-Cancer Agent
}

\author{
Lorenz Kirscher1, Xosé Luis Deán-Ben ${ }^{4}$, Miriam Scadeng 3 , Angelika Zaremba4, Qian Zhang², Christina \\ Kober ${ }^{1}$, Thomas Felix Fehm ${ }^{4}$, Daniel Razansky ${ }^{4}$, Vasilis Ntziachristos ${ }^{4}$, Jochen Stritzker ${ }^{1,2,}$, , Aladar A. \\ Szalay $1,2,5$, 冈 \\ 1. University of Würzburg, Department of Biochemistry, Am Hubland, 97074 Würzburg, Germany \\ 2. Genelux Cooperation, San Diego Science Center, 3030 Bunker Hill St, San Diego, CA 92109, USA \\ 3. University of San Diego, Center of Functional MRI, 9500 Gilman Drive, La Jolla, CA 92093, USA \\ 4. Helmholtz Institute, IBMI, Ingolstädter Landstraße 1, 85764 Oberschleißheim, Germany \\ 5. Department of Radiation Oncology, Moores Cancer Center, University of California, La Jolla, CA 92093, USA
}

\begin{abstract}
$\triangle$ Corresponding authors: JS (js@genelux.com; phone: +1-858-483-0024) and AAS (szalay@biozentrum.uni-wuerzburg.de; phone: +49-931-31 84410).
(C) 2015 Ivyspring International Publisher. Reproduction is permitted for personal, noncommercial use, provided that the article is in whole, unmodified, and properly cited. See http://ivyspring.com/terms for terms and conditions.
\end{abstract}

Received: 2015.04.28; Accepted: 2015.05.14; Published: 2015.06.15

\begin{abstract}
We reported earlier the diagnostic potential of a melanogenic vaccinia virus based system in magnetic resonance (MRI) and optoacoustic deep tissue imaging (MSOT). Since melanin overproduction lead to attenuated virus replication, we constructed a novel recombinant vaccinia virus strain (rVACV), GLV-1h462, which expressed the key enzyme of melanogenesis (tyrosinase) under the control of an inducible promoter-system. In this study melanin production was detected after exogenous addition of doxycycline in two different tumor xenograft mouse models. Furthermore, it was confirmed that this novel vaccinia virus strain still facilitated signal enhancement as detected by MRI and optoacoustic tomography. At the same time we demonstrated an enhanced oncolytic potential compared to the constitutively melanin synthesizing rVACV system.
\end{abstract}

Key words: Oncolysis, Molecular Imaging, Reporter gene, virotherapy

\section{Introduction}

Early detection of cancer facilitates early treatment and a better success rate for therapy. Consequently, a number of different applications to detect cancer cells are currently in development or already in daily clinical use. One option is the serological diagnosis of tumor biomarkers (e.g. alpha-fetoprotein (AFP) as a marker for hepatocellular carcinoma [1], prostate specific antigen (PSA) for prostate cancer [2] or the carcinogenic embryonic antigen (CEA) for different cancer types [3]). However, due to false-positive as well as false-negative results these serological tests require confirmation by tissue biopsies or direct imaging techniques. Imaging modalities that are currently used for cancer detection include computed tomography (CT), ultrasound, nuclear imaging modalities such as single-photon emission computer tomography (SPECT) and positron emission tomography (PET), as well as magnet resonance imaging (MRI). More recently (multispectral) optoacoustic tomography (MSOT) [4] is developed as another tool that will allow clinicians to detect tumors and their metastases $[5,6]$.

In an earlier publication we could show that melanin is an excellent reporter for both MRI as well as MSOT [7]. Melanin can be produced upon expression of the melanogenic enzyme tyrosinase (Tyr) and tyrosinase-related protein 1 (TYRP1) [7]. While the ability of melanin to bind free metal ions [8] leads to an intense MRI signal, presence of melanin also generated specific signals using MSOT due to its high absorption coefficient in the near infrared optical window $[7,9,10]$. It was shown that recombinant vac- 
cinia virus strains (rVACVs) encoding melanogenic enzymes had great theranostic potential [7]. However, the constitutive production of melanin resulted in decelerated viral replication of the melanogenic rVACV strains and therefore the therapeutic potential was diminished when compared to the non-melanogenic parental rVACV strain GLV-1h68.

Here, we enhanced the therapeutic potential of melanogenic rVACV by constructing a new melanogenic vaccinia virus strain (GLV-1h462) that encodes the melanogenic tyrosinase under control of an inducible promoter-system. We confirmed melanin production after exogenous induction in tumor bearing mice and analyzed the underlying kinetics. Furthermore, we could show that this new strain retained its imaging capabilities but at the same time had enhanced oncolytic potential compared to a constitutively melanin producing rVACV control strain. The synergistic effects of therapy and diagnosis of the melanogenic rVACV were therefore improved by taking advantage of the inducible promoter-system.

\section{Materials and Methods}

\section{Cell cultures and infection experiments}

The cell lines A549 and PANC-1 (obtained from the American Culture Collection) were cultured in RPMI 1640 (Sigma-Aldrich, Schnelldorf, Germany) and DMEM (Sigma-Aldrich), respectively, supplemented with $10 \%$ fetal bovine serum (FBS; PAA, Cölbe, Germany) and $1 \% 100 \times$ penicillin $(10000$ units $/ \mathrm{ml}) /$ streptomycin $(10 \mathrm{mg} / \mathrm{ml})$ (PAA). The cultures were incubated in a humidified incubator at $37^{\circ} \mathrm{C}$ and $5 \% \mathrm{CO}_{2}$ atmosphere.

One day prior to infection, A549 or PANC-1 cancer cells were seeded into 6 or 24 well plates to achieve $90 \%-95 \%$ confluence at the day of infection. The cells were infected with different rVACVs at different MOIs in infection medium (RPMI 1640 or DMEM $+2 \%$ FBS and 1\% penicillin/streptomycin). After incubation for $1 \mathrm{~h}$, the infection medium was replaced with fresh growth medium (RPMI 1640 or $\mathrm{DMEM}+10 \% \mathrm{FBS}+1 \%$ penicillin/streptomycin).

\section{Construction of an rVACV strain with induci- ble tyrosinase expression}

The codon optimized tet $R$ sequence was amplified with primers tetR-5 and tetR-3 from the template FH1tUTG [11] (courtesy of Dr. M. Herold), and the PCR product was cloned into the pCRII-Blunt-TOPO vector (Invitrogen). All primer and promoter sequences are listed in Supplementary Table S1. After sequence verification, the tet $R \mathrm{cDNA}$ was cut out with Xho I and BamH I, and ligated into pSC65 [12] with the same cuts, thus placing the tetR gene under the control of vaccinia virus promoter $\mathrm{p7.5}$. The $\mathrm{p} 7.5$-tet $R$ expression cassette was released by Hind III and BamH I, and subsequently put into the vaccinia virus transfer vector for F14.5L locus as in the final construct Fp7.5-tetR. The tetR cDNA under the control of vaccinia virus strong synthetic early/late promoter (SEL) was constructed by ligating the Xho I/Sal I fragment of tetR from Fp7.5-tetR to a FSEL vector precut with Sal I. The Fp7.5-tetR and FSEL-tetR were used to introduce tet $R$ gene into the virus genome.

To add tet $O$ to $C B G-2 A-m R F P$ and $m$ Tyr, respectively, the primer pairs tetO-mRFP-5 / mRFP-3 and tetO-mTyr-5 / mTyr-3 were used to amplify the target cDNAs from constructs containing CBG-2A-mRFP and mTyr. The PCR products were cloned into the pCRII-Blunt-TOPO vector. The tetO-CR (te$t O-C B G-2 A-m R F P)$ and tetO-mTyr cDNAs were released by Sal I and Pac I from pCRII-tetO-CR and pCRII-tetO-mTyr, and ligated to the vaccinia virus transfer vectors for the TK locus with the same cuts, placing the tetO containing cDNAs under the control of vaccinia virus strong synthetic late promoter SL resulting in TK-SL-tetO-CR and TK-SL-tetO-mTyr, which were used for the generation of recombinant viruses.

All virus strains were constructed using a selection process that is dependent on the expression of guanine phosphoribosyltransferase [13].

\section{Virus strains}

The tyrosinase-negative strains GLV-1h68 [14] and GLV-1h312 [15] were used as non-melanogenic control viruses. The strain GLV-1h460 encodes the main melanogenic enzyme $m T y r$ under control of the synthetic late promoter followed by the tet-operator sequence tet $O$. Due to the absence of the tet-repressor TetR, the expression is not inhibited and occurs in the late phase of the viral replication cycle. In contrast, GLV-1h462 encodes TetR under control of the synthetic early-late promoter, which should lead to the presence of the regulator protein during the whole infection cycle. Consequently, the mTyr expression should only occur in the presence of doxycycline, when TetR dissociates from the tet-operator sequence [16].

\section{Western blot analysis}

A549 cancer cells were infected with GLV-1h312, GLV-1h460 and GLV-1h462 respectively, at an MOI of 0.1 in the presence or absence of doxycycline (1 $\mu \mathrm{g} / \mathrm{ml})$. After one day, the medium was aspirated and the cell layer lysed in SDS sample buffer, transferred to fresh $1.5 \mathrm{ml}$ tube and heated to $99^{\circ} \mathrm{C}$ for $10 \mathrm{~min}$. Protein lysates were separated by $10 \%$ SDS-Polyacrylamide gel electrophoresis and trans- 
ferred onto a nitrocellulose membrane (Whatman $\mathrm{GmbH}$, Dassel, Germany). After blocking, the membrane was incubated with anti-human-tyrosinase goat polyclonal antibody (sc-7833, Santa Cruz Biotechnology Inc, Santa Cruz, USA), anti-beta-glucuronidase rabbit polyclonal antibody (G5420, Sigma-Aldrich), anti-beta-actin mouse monoclonal antibody (ab6276, Abcam, Cambridge, UK), anti-RFP rabbit polyclonal antibody (AB231, Evrogen, Moscow, Russia), anti-GFP rabbit polyclonal antibody (sc-8334, Santa Curz Biotechnology Inc) and anti-TetR mouse monoclonal antibody (Clone 9G9, Clontech, Mountain View, USA) respectively. The protein bound primary antibodies were detected using horseradish peroxidase conjugated rabbit anti-mouse (ab6728, Abcam), goat anti-rabbit (ab6721, Abcam) or rabbit anti-goat (ab6741-1, Abcam) secondary antibodies, followed by enhanced chemiluminescence detection with the molecular imager ChemiDox XRS+ from Bio Rad (Bio Rad, Munich, Germany).

\section{Cytotoxicity assay}

A549 or PANC-1 tumor cells were seeded in 24 well plates and infected at $\sim 95 \%$ confluence with an MOI of 0.01 (A549) or MOI 0.1 (PANC-1). At each point, supernatant was discarded and cell layer covered with $250 \mu \mathrm{l}$ of a $2.5 \mathrm{mg} / \mathrm{ml}$ Methylthiazolyldiphenyl-tetrazolium bromide (MTT; M2128, Sigma-Aldrich) solution. After incubation for $2 \mathrm{~h}$ at $37^{\circ} \mathrm{C}$ the supernatant was discarded, the formazan crystals resolved in $1 \mathrm{~N} \mathrm{HCl}$ /isopropyl alcohol and the optical density at $570 \mathrm{~nm}$ determined. Afterwards, the relative survival was calculated related to uninfected controls, which were considered as $100 \%$ at each time point.

\section{Viral replication assay}

To compare the replication of GLV-1h462 in the presence or absence of doxycycline $(1 \mu \mathrm{g} / \mathrm{ml})$ a standard plaque assay was performed. Therefore, A549 or PANC-1 tumor cells were seeded into a 24 well plate, incubated to a confluence of $\sim 95 \%$ and infected with the non-melanogenic rVACV GLV-1h68 as a control and the inducible melanogenic GLV-1h462 at MOI 0.01 (A549) or MOI 0.1 (PANC-1). One triplicate of each group at each time point was incubated with $1 \mu \mathrm{g} / \mathrm{ml}$ doxycycline while one was incubated without doxycycline. 24, 48 and 72 hours post viral infection (hpvi) samples were collected and stored at $-20^{\circ} \mathrm{C}$. A standard plaque assay [17] was performed to obtain the viral titers (plaque forming units per milliliter $[\mathrm{pfu} / \mathrm{ml}]$ ) at each time point.

\section{B-gucuronidase assay}

All rVACV strains in this study encode the Escherichia coli ß-glucuronidase gene gusA. The gene product is not secreted and released only upon cell lysis. Therefore, the concentration of glucuronidase in the blood can be used as a marker for viral replication and oncolysis. The B-glucuronidase activity can be determined as previously reported [18].

\section{Plaque Size measurements}

A549 lung carcinoma cells, PANC-1 human pancreatic tumor cells or CV-1 cells were infected with the upon induction tyrosinase expressing GLV-1h462, the constitutively tyrosinase expressing GLV-1h460 and the non-melanogenic GLV-1h312. After $1 \mathrm{~h}$ infection the cells were covered with medium containing $1.5 \%(\mathrm{w} / \mathrm{v})$ carboxymethylcellulose (Sigma-Aldrich) with or without doxycycline $(1 \mu \mathrm{g} / \mathrm{ml})$. In some experiments, medium containing carboxymethylcellulose with doxycycline was replaced with the same medium without dox after a single wash with $1 \mathrm{ml}$ PBS. After two or three days of infection cell layers were stained with crystal violet and plaques photographed with an Axiovert $200 \mathrm{M}$ microscope equipped with a digital AxioCam MRc5 (Zeiss, Jena, Germany) camera and the Axiovision 4.5 software. For each group, the maximal diameters of 25 to 104 plaques were determined using Image J $1.47 \mathrm{v}$.

\section{Blood sample collection}

To determine the virus mediated oncolysis by measuring the E. coli B-glucuronidase activity, blood was taken 56 days post rVACV injection. Whole blood was collected in BD Microtainer SST tubes (BD Bioscience, Heidelberg, Germany) and allowed to coagulate on ice, followed by a centrifugation step at 13000 rpm for $20 \mathrm{~min}$ at $4^{\circ} \mathrm{C}$ to separate blood cells from serum. The serum was stored at $-80^{\circ} \mathrm{C}$ until usage.

\section{Animal studies}

A549 or PANC-1 xenograft tumors were developed in 6- to 8- week-old female or male nude mice (Athymic Nude Foxn1nu, Harlan, Borchem, Germany and Livermore, USA) by implanting $5 \times 10^{6}$ A549 cells or $4 \times 10^{6}$ PANC- 1 cells subcutaneously in the right hind flank. Fourteen days after A549 tumor cell implantation, a single dose of PBS, or $5 \times 10^{6}$ plaque forming units (pfu) of a not tyrosinase expressing control virus strain, GLV-1h460 or GLV-1h462 in 100 $\mu \mathrm{L}$ of PBS was injected into a lateral tail vein of mice. PANC-1 xenograft bearing athymic nude mice were systemically injected under isoflurane narcosis by administration of $100 \mu \mathrm{rVACV}$ suspensions into the retro-orbital sinus vein at an average tumor volume of about $200 \mathrm{~mm}^{3}$. Weights of the mice and tumor growth, in two dimensions using a digital caliper, were recorded once a week. Tumor volume was calculated with the formula ([length $\times$ width $\left.^{2}\right] \times 0.52$ ). For induction of tyrosinase expression in GLV-1h462 injected mice, doxycycline was supplied in the 
drinking water $(100 \mu \mathrm{g} / \mathrm{ml}$ doxycycline) or daily via intraperitoneal injection (i.p.) of $100 \mu \mathrm{g} /$ mouse.

\section{Ethics Committee Approval}

All animal experiments were carried out in accordance with protocols approved by the Institutional Animal Care and Use Committee (IACUC) of Explora Biolabs (San Diego, USA, protocol number EB11-025) or the government of Unterfranken (Würzburg, Germany, protocol numbers AZ 55.2-2531.01-17/08 and 55.2-2531.01-76/13).

\section{L-DOPA turnover rate}

To compare the activity of mTyr the substrate (L-DOPA) turnover was measured over time by increase of absorbance at $475 \mathrm{~nm}$. A modified method of Hida et al. was used [19]. A549 cancer cells were infected with the different rVACV strains at an MOI of 0.1 . One group was supplemented with $1 \mu \mathrm{g} / \mathrm{ml}$ doxycycline while no doxycycline was added to the other. Twenty-four hours later, the supernatant was aspirated and the cells lysed in phosphate lysis buffer (32.5mM Na $2 \mathrm{HPO}_{4}-2 \mathrm{H}_{2} \mathrm{O}, 34,2 \mathrm{mM} \mathrm{KH}_{2} \mathrm{PO}_{4}, 1 \%$ TritonX, $\mathrm{pH}$ 6.8), transferred to a fresh $1.5 \mathrm{ml}$ tube and centrifuged (10000 rpm) to remove cell debris. Protein concentrations were determined with standard Bradford assay. From each normalized sample $180 \mu \mathrm{l}$ of supernatant was transferred to a 96 well plate and co-incubated with $20 \mu \mathrm{l}$ of a $10 \mathrm{mM}$ L-DOPA (Sigma-Aldrich) solution. The increase in absorbance at $475 \mathrm{~nm}$ was measured for $2 \mathrm{~h}$ every $10 \mathrm{~min}$ at room temperature in an absorbance reader (Tecan Sunrise, Mannedorf, Switzerland). The mean velocity (v) was defined as increase of optical density at $475 \mathrm{~nm}$ $\left(\mathrm{OD}_{475 \mathrm{~nm}}\right)$ per time interval (0 - 120min).

\section{Determination of melanin amounts in cell cultures and tumor tissues}

\section{Cell cultures}

The melanin content of different samples was determined by using a modified protocol of Hida et al. [19]. Infected A549 or PANC-1 cancer cells were trypsinized (1xTyrpsin + EDTA; PAA) until cells detached. Trypsinization was stopped with growth medium (total volume $1 \mathrm{ml}$ ), transferred to a fresh $1.5 \mathrm{ml}$ tube and centrifuged (13000 rpm). Afterwards, the cells were washed with HBSS (PAA) followed by another centrifugation (13000 rpm) step. The supernatant was discarded, the pellet resuspended in $1 \mathrm{M}$ $\mathrm{NaOH}$ and heated to $99^{\circ} \mathrm{C}$ for $10 \mathrm{~min}$. After cooling down, $100 \mu$ of each sample was transferred into a 96 well plate and the $\mathrm{OD}_{475 \mathrm{~nm}}$ was determined in an absorbance reader (Tecan Sunrise).

\section{Quantification of melanin using (multispectral) optoacoustic tomography (MSOT)}

Tumor Xenografts

Volumetric (three dimensional) MSOT (vMSOT) images of the excised tumors were taken ex vivo. The vMSOT system described in detail in [20] allows fast (high-throughput) imaging of the whole tumor volume by simultaneous detection of signals with 256 sensors distributed on a spherical surface. Tumor images were acquired at 740, 760 and $780 \mathrm{~nm}$, and subsequently averaged 200 times at each wavelength for noise reduction. The reconstructed three-dimensional images represent the optical absorption at each wavelength with an almost isotropic resolution of $200 \mu \mathrm{m}$. The distribution of melanin specific absorption was then estimated by linearly un-mixing the multispectral images to a combination of melanin and deoxygenated hemoglobin. Indeed, the imaging wavelengths were chosen for efficiently un-mixing these two chromophores with known optical absorption spectra. Because the melanin absorption is proportional to its concentration, the amount of melanin can be deduced from the un-mixed images.

\section{Tumor tissue homogenates}

Homogenates of these tumors were placed into an agarose phantom and measured at $700 \mathrm{~nm}$. Optoacoustic signal measurements were performed at different positions of the sample. The observed region consisted of 24 individual slices and had a total length of $11.5 \mathrm{~mm}$. In a second position of the agarose phantom a tumor tissue homogenate containing no melanin was measured in parallel serving as background control. Optoacoustic signals over background were measured at a single wavelength of 700 $\mathrm{nm}$ for each slice. The total signal per region of interest (ROI) was calculated and arithmetically averaged for each sample.

All experiments that were performed in this study were below the safety exposure limit for laser radiation in the near-infrared, which is $20 \mathrm{~mJ} / \mathrm{cm}^{2}$ for $700 \mathrm{~nm}$, and even higher at higher wavelength.

\section{Magnetic Resonance Imaging (MRI)}

Tumor homogenates were imaged on a 7 Tesla small animal imaging system (Bruker BioSpec, Billerica, USA). T1 relaxation time maps (rapid acquisition with refocused echoes [RARE]-T1+T2-map saturation recovery TR/TE, 5000-200/11-99ms, Field of view 5.5 $\mathrm{cm}$, matrix $256 \times 256$, slice thickness $1 \mathrm{~mm}, 8$ slices interleaved, total imaging time $19 \mathrm{~min} 43$ seconds) were acquired on 2-4 slices through sealed straws containing tumor homogenates. The straws were embedded in low melting agarose (5\% in PBS) to prevent surface artifacts. The T1-values of each slice 
were averaged from the calculated T1-values in each pixel of a manually drawn region of interest, which included the interior of the straw cross-section. R1-relaxation rates were calculated from the obtained $\mathrm{T} 1$ values (R1-relaxation rate $=1 / \mathrm{T} 1$ value).

\section{Statistical analyses}

A two-tailed Student's $t$ test was used for statistical analysis. A $p$ value of $\leq 0.05$ was considered statistically significant.

\section{Results}

\section{rVACV marker gene expression in infected A549 lung carcinoma cells}

The vaccinia virus strains GLV-1h312, GLV-1h460 and GLV-1h462 (Fig. 1A) were tested for expression of the inserted marker genes. For this, A549 lung carcinoma cells were infected with these different rVACVs using an MOI of 0.1. Protein samples were collected after an incubation time of $24 \mathrm{~h}$ in presence or absence of doxycycline $(1 \mu \mathrm{g} / \mathrm{ml})$ and analyzed by Western blot (Fig. 1B). Human beta-actin which served as a loading control was detected in each lysate. As expected, expression of the $25 \mathrm{kDa}$ Tet-repressor could be detected in GLV-1h312 and GLV-1h462 infected cells, in the presence or absence of doxycycline (dox), while Ruc-GFP (64 kDa) was found only in GLV-1h460 infected A549 cells (Fig. 1B). The functionality of the tet-system was confirmed as well. Expression of regulated genes could only be detected in the presence of dox, when the conformational change of TetR resulted in dissociation from the tet-operator allowing transcription of the viral promoter regulated expression cassette by the viral RNA-polymerase. The mRFP1 molecule $(30 \mathrm{kDa})$ inserted into the TK locus of GLV-1h312, was detected solely in GLV-1h312 infected A549 cancer cells in presence of dox. GLV-1h462 infected A549 cells showed $m$ Tyr $(70 \mathrm{kDa})$ expression in presence of dox

A

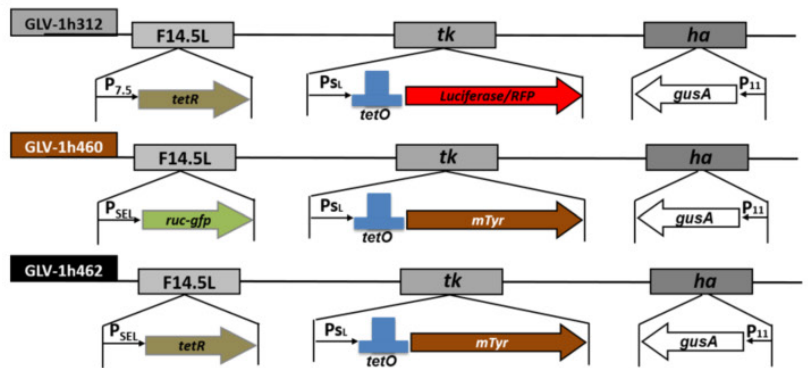

but not when the inducer was absent. Lung carcinoma cells infected with GLV-1h460, which does not encode tet $R$, produced mTyr both in the absence or presence of dox. All three rVACV strains lead to expression of GusA, the E. coli beta-glucuronidase $(60 \mathrm{kDa})$, inserted in the viral HA locus (Fig. 1A) in presence or absence of dox (Fig. 1B). Mock infected A549 cells served as negative control for the virus encoded marker proteins.

\section{Melanin synthesis in melanogenic rVACV in- fected A549 cancer cells}

To find the dox concentration at which mTyr expression was induced, GLV-1h462 infected A549 cancer cells were incubated in the presence of increasing dox concentrations $(0,0.01,0.05,0.1,0.5$ and 1 $\mu \mathrm{g} / \mathrm{ml}$ ) (Fig. 2A). Mock infected and GLV-1h312 infected A549 lung carcinoma cells served as a negative control for melanin production and showed low background levels as indicated by optical density measurements at $475 \mathrm{~nm}\left(\mathrm{OD}_{475}\right)$. Non-infected A549 cells had an almost constant $\mathrm{OD}_{475}$ of about $0.1 \pm 0.01$ regardless of the applied dox concentration. The somewhat lower $\mathrm{OD}_{475}$ of GLV-1h312 infected cells compared to mock infected cells can be explained by reduced cell numbers due to viral infection. In case of GLV-1h460 infected A549 cells, which produced melanin in a constitutive manner (Fig. 1A and B), a nearly constant high absorbance of $0.28 \pm 0.01$ at 475 $\mathrm{nm}$ could be detected regardless of the amounts of dox in the medium. In contrast, GLV-1h462 infected A549 cancer cells showed a low optical density at a dox concentration of $0 \mu \mathrm{g} / \mathrm{ml}(0.087 \pm 0.003)$ and 0.01 $\mu \mathrm{g} / \mathrm{ml}(0.103 \pm 0.014)$. At a dox level of $0.05 \mu \mathrm{g} / \mathrm{ml} \mathrm{a}$ significant increase $(\mathrm{p}<0.001)$ in $\mathrm{OD}_{475}(0.284 \pm 0.009)$, and therefore melanin synthesis, was detected. This dox concentration $(0.05 \mu \mathrm{g} / \mathrm{ml})$ seemed to saturate the inducible system in vitro as no further increase in $\mathrm{OD}_{475}$ with higher dox concentrations was observed.

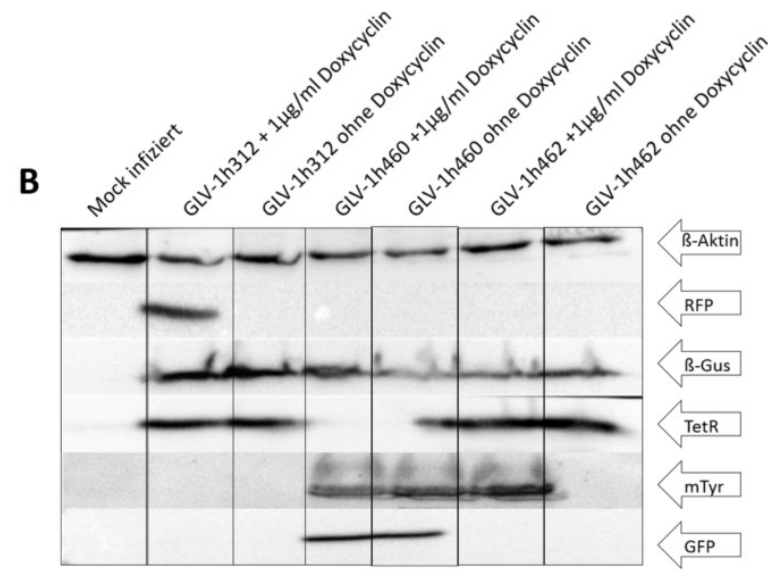

Figure 1: rVACV mediated expression of proteins in A549 cancer cells. A) Map of relevant insertions into the genome of the different rVACV strains. B) Western blot analysis of viral marker (TetR, RFP, GusA, Ruc-GFP, mTyr) expression in rVACV infected A549 lung carcinoma cells in presence or absence of dox. 
In addition to the absorbance measurements, the velocity of L-DOPA turnover, as a measure of tyrosinase activity, of GLV-1h460 and GLV-1h462 infected A549 cells 24 hours post virus infection (hpvi) was determined (Fig. 2 B). While lysates of GLV-1h460 infected A549 cancer cells showed no significant difference in mean velocity $(v)$ in the presence $(0.089 \pm$ $\left.0.010 \Delta \mathrm{OD}_{475} / 10 \mathrm{~min}\right)$ or absence $(0.091 \pm 0.010$ $\Delta \mathrm{OD}_{475} / 10 \mathrm{~min}$ ) of dox, cell lysates of GLV-1h462 infected A549 cells showed a significantly ( $\leq \leq 0.01$ ) higher L-DOPA turnover rate in presence $(0.074 \pm$ $\left.0.006 \Delta \mathrm{OD}_{475} / 10 \mathrm{~min}\right)$ than in absence $(0.000 \pm 0.000$ $\Delta \mathrm{OD}_{475} / 10 \mathrm{~min}$ ) of dox.

\section{Enhanced spreading capacity of non-induced not tyrosinase expressing rVACV GLV-1h462}

Constitutive production of melanin resulted in reduced viral replication as well as decelerated tumor regression when compared to non-melanogenic rVACV [7]. To analyze whether we can achieve better replication and spreading in the non-induced state, A549 cells were infected with GLV-1h460 and GLV-1h462 in the presence or absence of $1 \mu \mathrm{g} / \mathrm{ml}$ dox (Fig. 3A and B). Plaques of non-induced and hence not melanin synthesizing GLV-1h462 were bigger than plaques of constitutively melanin producing GLV-1h460 or those of dox-induced melanogenic GLV-1h462 (Fig. 3A). Quantitative analysis of the maximum plaque diameters of each group (Fig. 3B) revealed that the maximum plaque diameters of non-induced not melanin producing GLV-1h462 infected A549 cells were significantly $(\mathrm{p}<0.001)$ bigger (maximum diameter: $124 \pm 32 \mu \mathrm{m}$ ) than those of induced melanin synthesizing GLV-1h462 infected cells $(71 \pm 31 \mu \mathrm{m})$ or GLV-1h460 infected A549 in the absence $(67 \pm 24 \mu \mathrm{m})$ or presence $(62 \pm 22 \mu \mathrm{m})$ of dox. No significant plaque-diameter difference between mela- nin producing plaques were observed. Similar results were observed in PANC-1 tumor cells (data not shown). In addition to the enhanced spreading capacity a significantly better oncolytic potential in A549 (Fig. 3C) at 48, 72 and 96 hpvi and in PANC-1 (Fig. 3D) cells at 72 and 96 hpvi of not induced and not melanin-producing GLV-1h462 was demonstrated. Furthermore, a higher replication rate (Supplementary Fig. S1) was observed.

\section{Doxycycline exposure inversely correlates with oncolytic potential of GLV-1h462}

In a clinical setting, the best synergistic effects of the diagnostic and the therapeutic potential of the melanogenic rVACV should be achieved when the production of melanin does not interfere with the oncolytic potential or is reduced to a minimum. Since constitutive melanin production obviously reduced the oncolytic potential (reduced plaque size upon dox induction in Fig. 3A, B), it was necessary to evaluate the therapeutic effects of the virus in a setting of transient doxycycline exposure. It was expected that the shorter the exposure to doxycycline, the higher the oncolytic potential would be.

Cells were therefore infected with a defined number of plaque forming units of GLV-1h462 and control strains respectively. The doxycycline containing overlay medium that was replaced with dox-free medium at different times post viral infection (at 0, 2, 6, 8, 24, 48 and 72 hpvi respectively). Consequently, tyrosinase (and therefore melanin)-production was transient. The infection was stopped simultaneously at 72 hours post viral infection and the maximum plaque diameter for at least 25 plaques/virus strain and dox-exposure time was measured (Fig. 4).
A

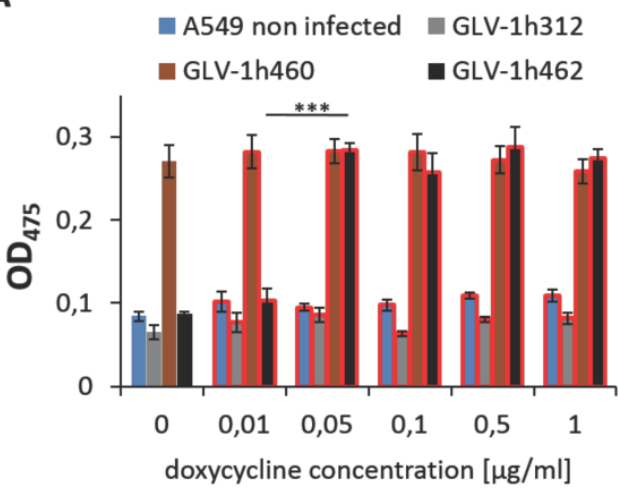

B

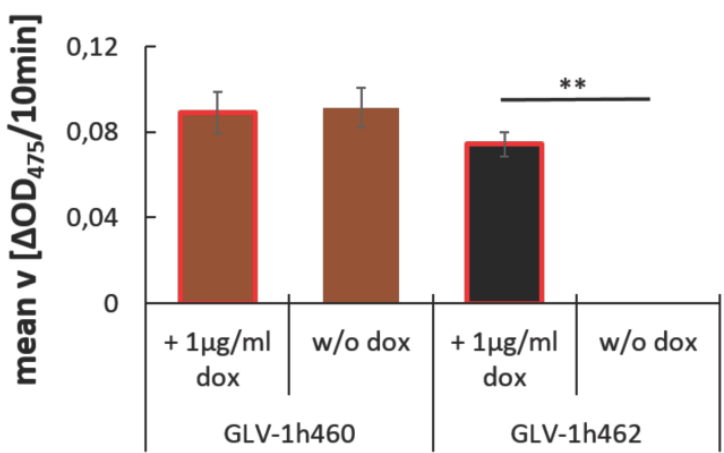

Figure 2: Induction of tyrosinase expression in GLV-1 h462 infected A549 cells A) Absorbance measurements at $475 \mathrm{~nm}$ of cell lysates from rVACV infected A549 cells incubated with different dox concentrations. Significantly higher optical density at $475 \mathrm{~nm}$ was obtained from GLV- $1 \mathrm{~h} 462 \mathrm{infected} \mathrm{A549} \mathrm{cells} \mathrm{at} \mathrm{a} \mathrm{concentration} \mathrm{of} 0.05 \mu \mathrm{gg} / \mathrm{ml}$ compared to $0.01 \mu \mathrm{g} / \mathrm{ml}$ doxycycline. No significant influence of dox on OD $475 \mathrm{~nm}$ of GLV-1h460, GLV-1h312 or mock infected A549 cancer cells. B) Mean velocity of L-DOPA turnover in A549 cell lysates from cells infected with melanogenic rVACV in presence or absence of dox. Significantly higher velocity of L-DOPA turnover in dox induced than non-induced GLV-1h462 infected A549 cells $(p<0.01)$. No significant dox-dependent difference in velocity in GLV-1h460 infected cells. 
A

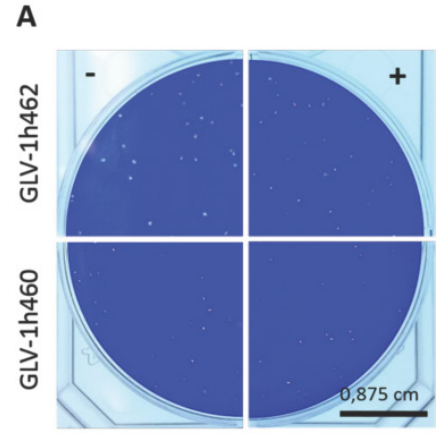

B

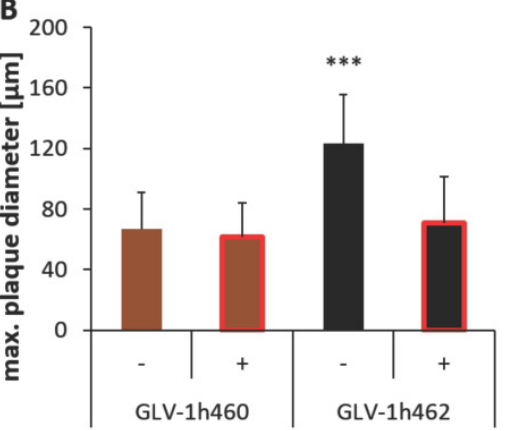

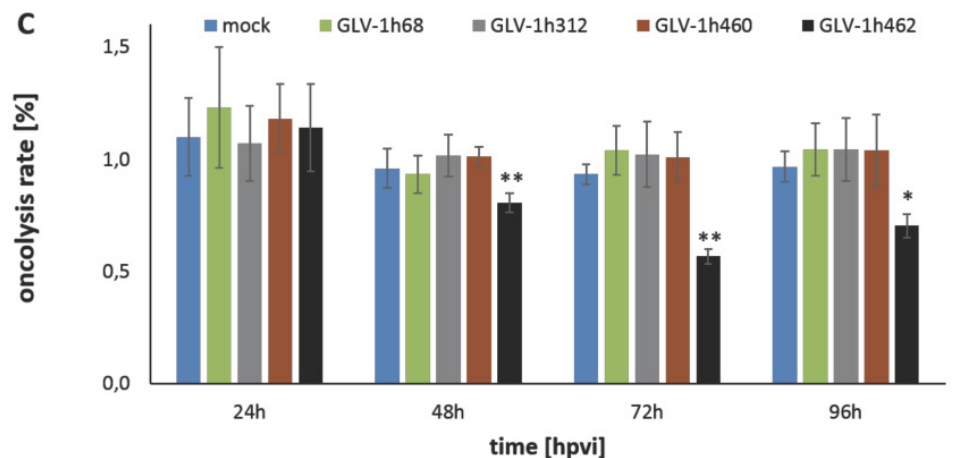

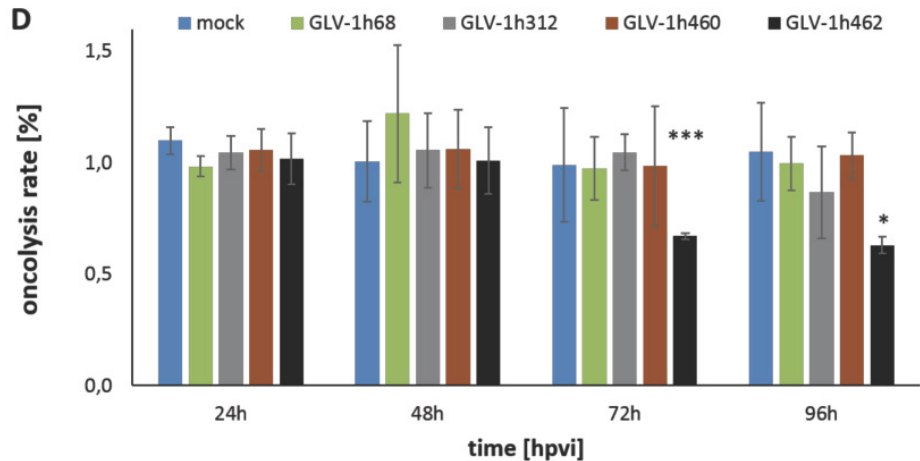

Figure 3: Oncolytic activity of inducible melanogenic rVACV strains. Photography A) and quantitative evaluation B) of plaque sizes in A594 cancer cells infected with melanogenic rVACV incubated in presence $(+)$ or absence $(-)$ of $1 \mu \mathrm{g} / \mathrm{ml}$ dox. Significantly larger mean maximum plaque diameter of non-induced not melanin producing GLV-1h462 infected A549 cancer cells compared to induced melanogenic GLV-1h462 infected A549 cells or GLV-1h460 infected A549 cancer cells ( $<0.001 ; n \geq 50$ ). MTT-Assay of A549 C) and PANC-1 D) cancer cells infected (MOI 0.01 or MOI 0.1) with the constitutively melanin synthesizing GLV-1h460, the non-melanogenic GLV-1h68 and GLV-1h312 and the dox inducible melanogenic GLV-1h462. The control rVACV showed no significant difference in oncolysis, regardless of absence or presence of doxycycline $(1 \mu \mathrm{g} / \mathrm{ml})$. In contrast, the inducible melanogenic GLV-1h462 infected groups showed a significantly higher oncolysis rate 48,72 and 96 hpvi in A549 cells and 72 and 96 hpvi in PANC-1 cells in the absence of dox $\left(* * * \mathrm{p}<0.001 ; *^{* *}<0.01\right.$, *p $\left.<0.5\right)$.

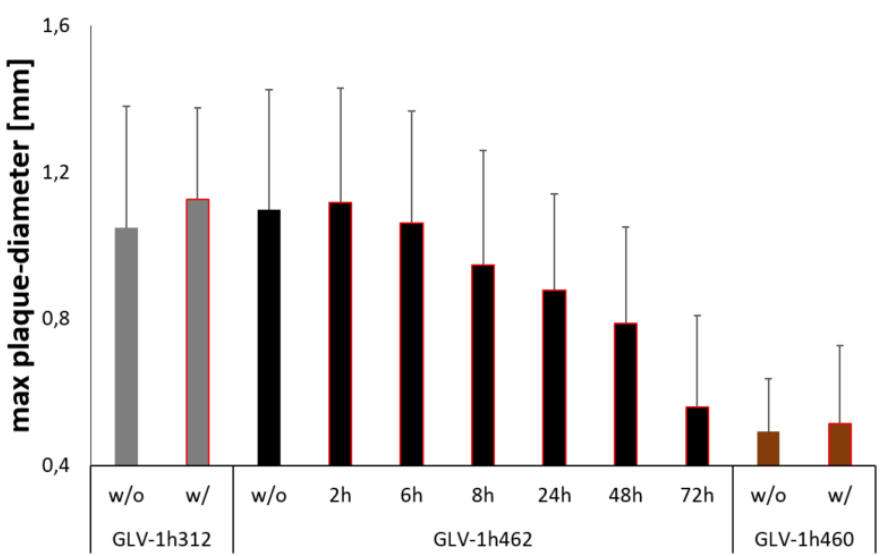

Figure 4: Effects of transient doxycycline induction on inducible melanogenic VACV based oncolysis. Quantitative evaluation of plaque sizes in CV-1 cells infected with inducible melanogenic GLV-1h462, constitutively melanogenic GLV-1h460 and non-melanogenic GLV- $1 \mathrm{~h} 312$ incubated in presence (w/) or absence (w/o) of $1 \mu \mathrm{gg} / \mathrm{ml}$ dox for 72 hours. CV-1 cells infected with GLV-1h462 were incubated for 2, 6, 8, 24, 48 and 72 hours with $1 \mu g / m l$ dox respectively. Data represent the average maximum plaque diameter plus standard deviation of at least 25 plaques/virus strain and dox exposure time. 


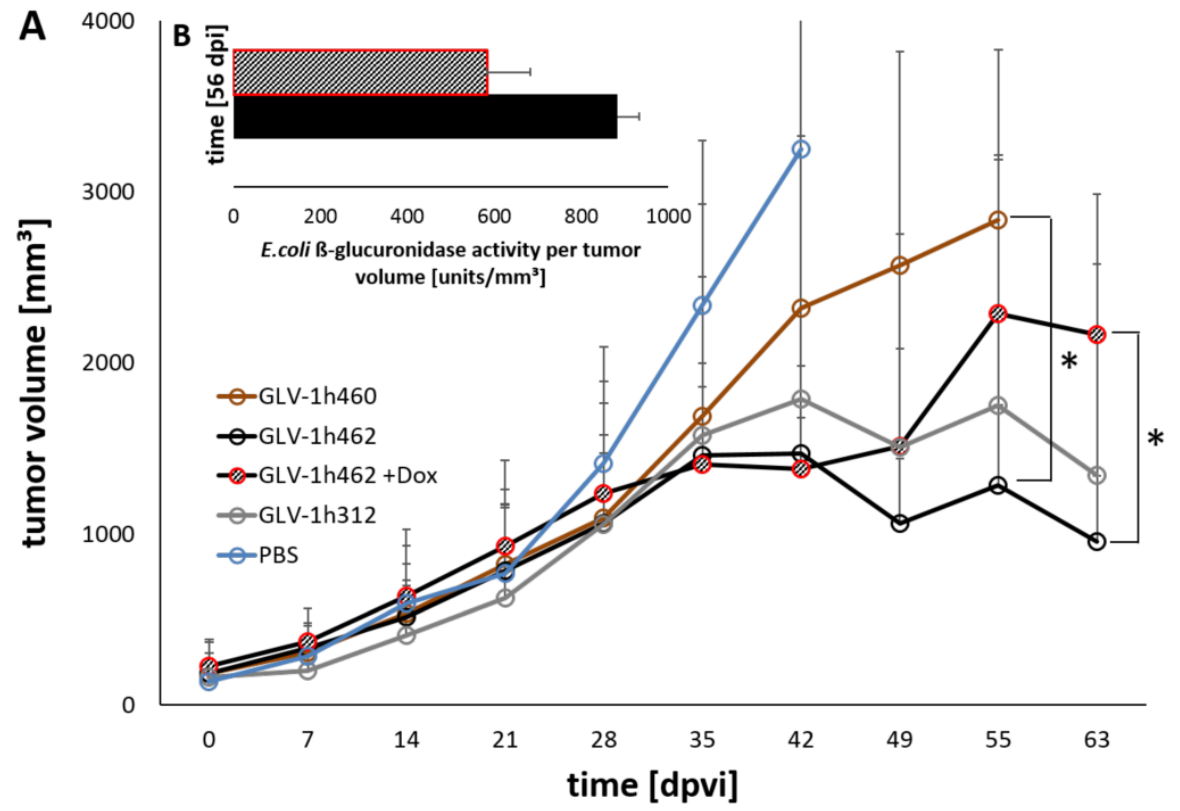

Figure 5: Viral activity and oncolytic potential of tested rVACV in PANC-1 xenograft bearing nude mice A) Tumor volume measurements of PANC-1 tumors untreated (PBS) or treated with GLV-1h460, GLV-1h312 and GLV-1h462 (n=3-5). Significant smaller tumor volume of uninduced non-melanogenic GLV-1h462 colonized PANC-1 tumors 55 dpvi compared to GLV-1h460 infected tumors and 63 dpvi compared to dox induced melanin synthesizing GLV-1h462 injected PANC-1 tumor bearing mice $(\mathrm{p}<0.05)$. B) Escherichia coli beta-glucuronidase activity assay of blood serum samples of PANC-1 xenograft bearing mice injected with GLV-1h462. GusA-activities are normalized by tumor volume as a correction factor.

As postulated, the negative effects on viral replication and spreading upon induction of melanin-synthesis were reversible. The longer the time interval without doxycycline induction the closer the therapeutic potential (max. plaque diameter) was to the control without dox or the non-melanogenic control strain (GLV-1h312). In this experiment co-incubation with dox for $8 \mathrm{~h}$ or more led to significant $(\mathrm{p}<0.05)$ smaller plaque diameters compared to GLV-1h462 infected cells, which were not incubated with dox or to dox treated GLV-1h312 control virus infected cells. When compared to the constitutively melanin producing vaccinia virus strain GLV-1h460, all plaques of GLV-1h462 infected cells showed significantly larger plaques, except the permanent $(72 \mathrm{~h})$ dox induced GLV-1h462 infected cells.

\section{Enhanced oncolytic potential of non-induced and therefore not melanogenic rVACV GLV-1h462}

Following the characterization in cell cultures we analyzed the effect of the different virus strains on xenograft tumor regression in mice. Therefore, nude mice bearing PANC-1 tumors $\left(\sim 200 \mathrm{~mm}^{3}\right)$ were injected with GLV-1h312, GLV-1h460 and GLV-1h462 respectively (Fig. 5). Five GLV-1h462 injected mice received $100 \mu \mathrm{g} / \mathrm{ml}$ dox in the drinking water while the other mice ( $\mathrm{n}=5$ per group) did not receive dox supplement. The tumor growth over time showed that the non induced GLV-1h462 infected tumors had a similar tumor volume as non-melanogenic
GLV-1h312 treated tumors. Also, the tumor volume in those mice decreased over time.

In contrast, the dox treated, melanin synthesizing GLV-1h462 injected PANC-1 xenograft bearing nude mice, exhibited higher tumor volumes, which were similar to those observed in the melanin producing GLV-1h460 injected group. Moreover, both groups showed a slightly increasing tumor volume, which resulted in a significant difference compared to the not induced GLV-h1462 injected group at day 55 post viral injection (dpvi) compared to GLV-1h460 injected mice and 63 dpvi concerning the induced melanin producing GLV-1h462 injected tumor bearing mice (Fig. 5A). A better therapeutic efficiency in uninduced GLV-1h462 colonized tumors was also observed in A549 tumor bearing mice (Supplementary Fig. S2A). Furthermore, the median tumor sizes of GLV-1h68 injected mice were very similar regardless of exposure to dox in this tumor model. Therefore, we concluded that dox had neither a negative influence on viral activity nor an anticancer effect at these concentrations.

To further investigate in vivo whether the viral replication and oncolysis of GLV-1h462 was improved if expression of the melanogenic enzyme mTyr was not induced, blood serum of PANC-1 tumor bearing mice infected with GLV-1h462 with or without induction with dox was collected. Subsequently, the E. coli beta-glucuronidase levels, as marker for viral replication [18], was determined (Fig. 5B). At 56 dpvi a difference between the E. coli be- 
ta-glucuronidase levels per $\mathrm{mm}^{3}$ tumor volume of non-induced GLV-1h462 injected mice in comparison to induced GLV-1h462 could be detected. Additionally to the blood sera of PANC-1 xenograft bearing mice, the beta-glucuronidase levels in blood sera of A549 tumor bearing mice was measured (Supplementary Fig. S2B). The amounts of enzyme per tumor volume at 49 and 56 dpvi were significantly higher in case of non-induced GLV-1h462 infected A549 tumor bearing mice $(p \leq 0.05)$. This indicated an increased oncolytic activity at these time points.

\section{Imaging of induced melanogenic rVACV with MRI and vMSOT technologies}

We reported earlier that rVACV dependent melanin production in infected tumor cells was visualized with magnetic resonance imaging (MRI) and multispectral optoacoustic tomography (MSOT) [7]. Here, we investigated if the dox inducible melanogenic reporter system GLV-1h462 is also suitable for both imaging technologies. Moreover, we wanted to
$2 d \quad 2 d \quad 4 d \quad 7 d$

(-)
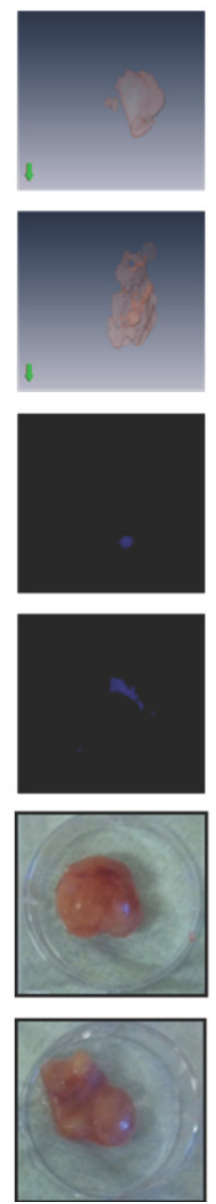

(+)
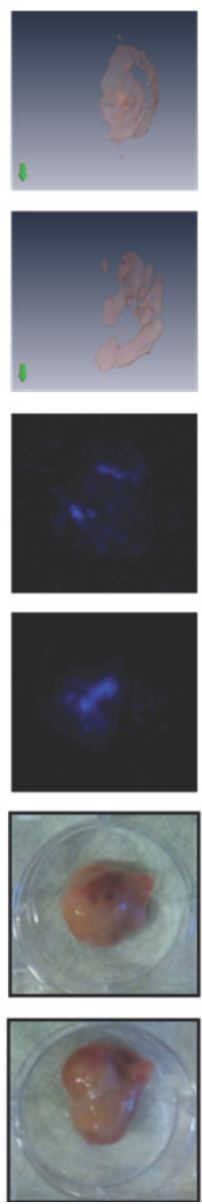

(t)
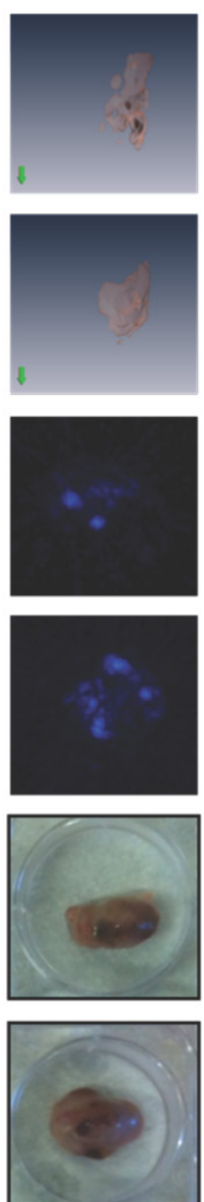

$(+)$
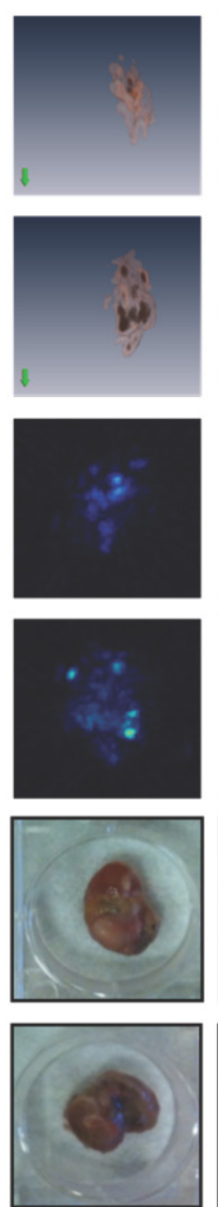

know how early melanin could be detected by either technology after dox induction in mice. We therefore excised A549 tumor xenografts at different times after the induction with dox and analyzed the MRI and volumetric MSOT (vMSOT) signals from these samples. Already 4 days post induction (dpi) clear melanin dependent vMSOT-signals were detected which were confirmed by visual inspections revealing the production of melanin (Fig. 6). Moreover, the melanin specific signals in vMSOT increased over time. The last time point measured (18dpi) showed the highest signal intensity while the non-induced GLV-1h462 infected A549 xenografts showed no or very little melanin specific signal. In PANC-1 tumors, we detected a melanin signal in GLV-1h460 and in induced GLV-1h462 colonized xenografts while no or very little melanin-dependent vMSOT-signal was observed in GLV-1h312 and uninduced GLV-1h462 tumors (Supplementary Fig. S3A).

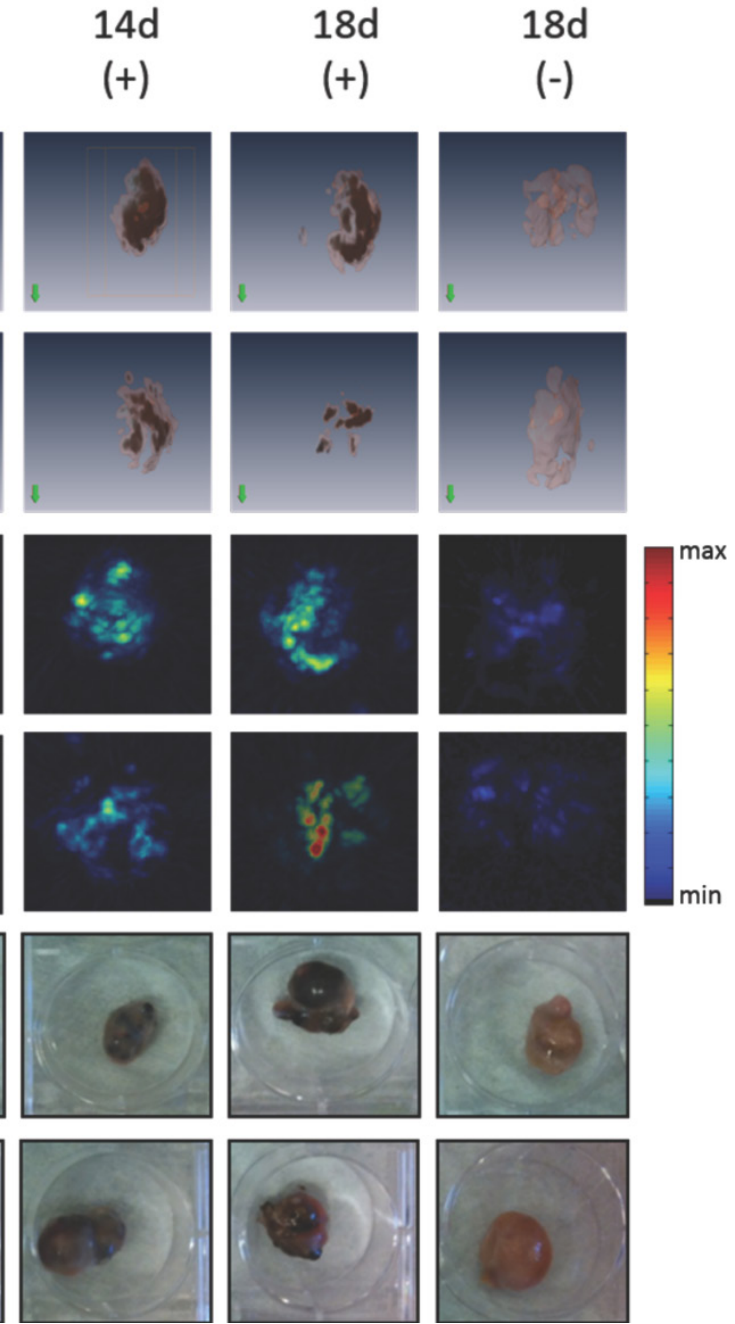

Figure 6: Ex vivo vMSOT-images and photographs of GLV-1 h462 infected A549 tumor xenografts with or without doxycycline induction. At each time point the same two excised A549 tumors of identical treatment are shown as 3D-reconstruction vMSOT images (upper 2 panels; pink represents tumor tissue; black represents melanin in tumor tissue), vMSOT maximum intensity projection images (middle 2 panels) and photographic pictures (lower 2 panels). Melanin specific signal intensity in the vMSOT images is presented as heat map. Left and right columns show the non-induced group (-), middle columns show tumors of doxycycline treated mice (+). Each column represents one time point after induction with doxycycline. The orientation of the tumors is not identical between the images. 
To further characterize the increasing melanin signal more quantitatively these tumors were homogenized and analyzed by MSOT and MRI (Fig. 7). In both cases, the received signals increased over time in the presence of dox for GLV-1h462 colonized tumor homogenates. The optoacoustic signal (Fig. 7A) of induced GLV-1h462 injected tumors was undistinguishable from the controls at the beginning of the dox induction and then increased steadily. Lysates of non-induced GLV-1h462 colonized tumors had optoacoustic signals that were in the range of the non-melanin producing controls even at the last time point. In contrast, lysates of GLV-1h460 colonized tumors contained much more melanin at the beginning of the experiment ( 2 days post start of the dox treatment). The somewhat higher melanin concentration at 18 days post dox treatment could be explained with the continuous production of melanin in those tumors.

A very similar observation was made for the R1 relaxation rates that were obtained from the very same lysates (Fig. 7B). The signal (R1 relaxation rate) increased over time in the GLV-1h462 containing samples upon dox induction.

Quantification of the melanin related max. MSOT signal intensities of PANC-1 tumors revealed significantly higher MSOT signal intensities in melanin synthesizing rVACV colonized tumors in comparison to non-melanin-producing vaccinia virus infected PANC-1 tumors (Supplementary Fig. S3B).

We also observed correlation in increase between the melanin dependent optoacoustic signal intensities and the increase in R1 relaxation rate obtained by MRI $\left(\mathrm{R}^{2}=0.49\right)$ (Fig. $7 \mathrm{C}$ ) which means melanin could be used adequately in both imaging modalities.

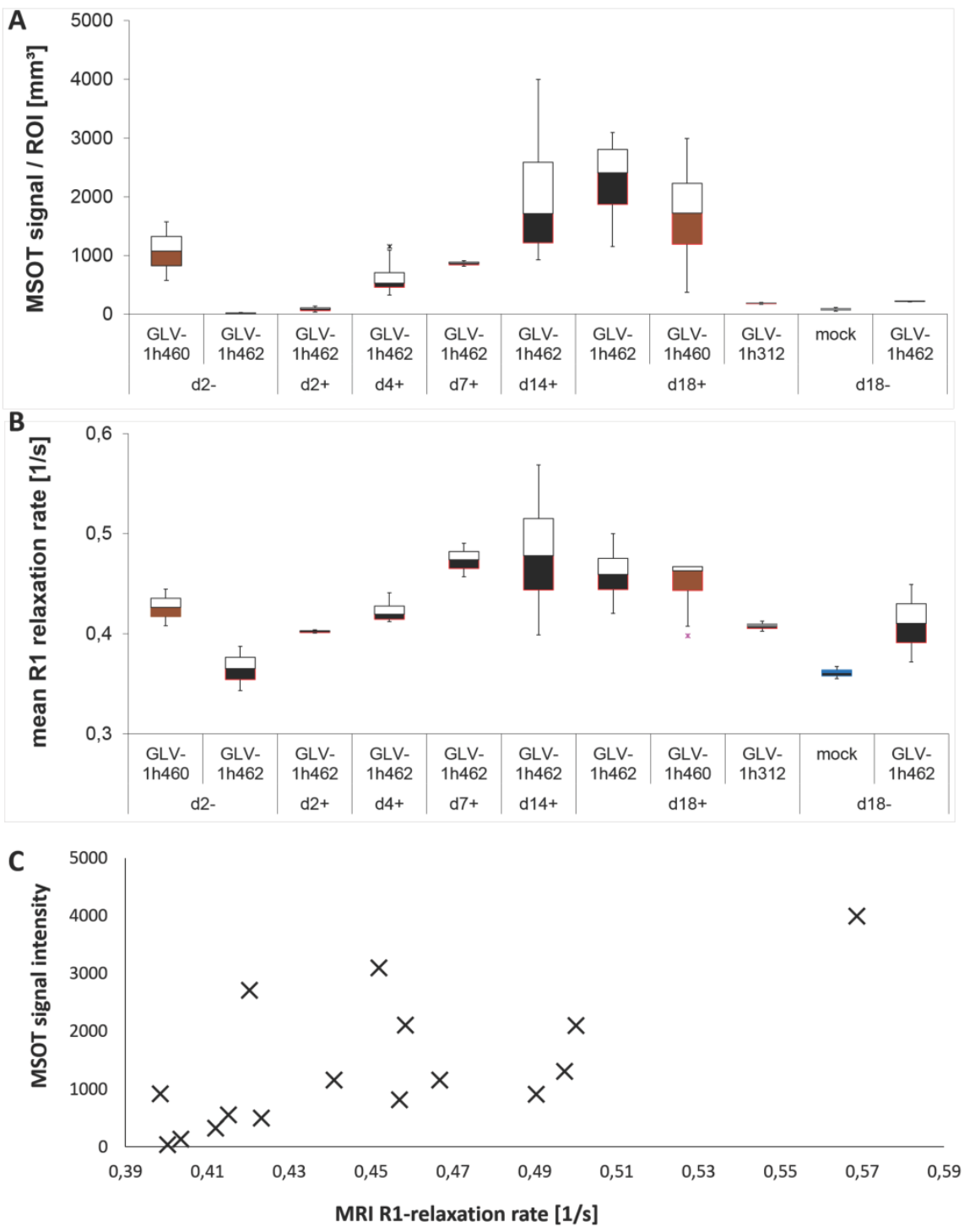

Figure 7: Evaluation of melanin amounts with MRI and optoacoustic imaging. Homogenized tissues of infected or PBS treated control A549 tumors analyzed by optoacoustic imaging at 700nm A) and MRI B) $(n=2-4)$. Correlation $C)$ of MRI and MSOT signal intensities of the values in A) and B) $\left(R^{2}=0,49\right)$. 


\section{Discussion}

Here, we showed that exogenously controlled melanin synthesis in vaccinia virus colonized tumor xenografts was feasible, resulting in improved synergistic effects of therapy and diagnosis. Doxycycline induction resulted in enhanced (v)MSOT and MRI signals. At the same time the therapeutic outcome of the inducible rVACV could be enhanced, when compared to constitutively melanin producing rVACV strains.

For induction of melanin production, we inserted the tet-regulated gene expression system components into the rVACV genome [15]. The system can be induced with doxycycline, a tetracycline derivate [21]. Doxycycline is a well-established and tolerated antibiotic [22]. Recent studies suggested an anti-cancer effect of tetracyclines due to inhibition of the matrix metalloproteinases (MMP) family. MMPs play an important role in tumor invasion and metastases formation by degradation of the tumor matrix. Additionally, MMPs are thought to activate tumor growth factors $\alpha$ and $\beta$ (TGF- $\alpha$ and $-\beta$ ), epidermal growth factor (EGF) and vascular endothelial growth factor (VEGF) [23]. In the study presented here, no difference concerning tumor volume between non-melanogenic GLV-1h68, in combination with doxycycline or GLV-1h68 injected mice not treated with doxycycline could be observed. Reduced levels of MMPs in the tumor tissue might lead to less tumor growth but it also reduces the viral distribution through a denser tumor matrix [24], which might compensate an adjuvant anti-cancer effect of doxycycline in combination with rVACV.

We have shown that the vaccinia virus construct GLV-1h462 leads to production of melanin in infected cell cultures and in mice but only after doxycycline exposure in A549 and PANC-1 cancer cells or tumors. The amounts of synthesized melanin in A549 tumors 4 days after induction was sufficient to generate a clear signal detectable with MRI or MSOT. We showed also that the amounts of melanin and therefore the signal intensities of both imaging modalities were increasing over time. Even though all of imaging experiments in this study were performed ex vivo, we do not have reason to believe that in vivo experiments would result in dramatic changes in signal intensities over background as we could show previously that melanin production can be detected in vivo by both MRI and MSOT [7].

Furthermore, we observed that plaque formation in induced melanin synthesizing GLV-1h462 infected tumor cells resulted in significantly smaller plaques than those of non-induced non-melanogenic GLV-1h462 infected cells, suggesting a greater onco- lytic potential. Indeed, non-induced GLV-1h462 had enhanced oncolytic effects compared to doxycycline-induced melanogenic GLV-1h462 in injected mice. The inhibition of $m T y r$ expression led to increased viral replication.

Previously, we observed that rVACV-mediated constitutive melanin production slowed down tumor regression [7]. Interestingly, Cunha et al. showed in cell cultures that due to melanogenesis in B16F10 murine melanogenic melanoma cell lines the levels of reactive oxygen species (ROS) increased, which led to cell cycle arrest [25]. The cell damage resulting from production of ROS might also slow down viral replication, either directly or through inhibitory effects in the host cell. Another reason for the reduced replication of melanogenic rVACV could be the depletion of L-tyrosine inside the cells due to the increased tyrosine consumption for virus-mediated melanin synthesis. Depletion of L-tyrosine consequently would reduce protein synthesis, which would negatively influence viral replication. While production of melanin can inhibit the growth of individual melanin producing cells, to our knowledge melanin itself has no systemic toxicity. Intermediates in melanin biosynthesis are reported to have toxicity, which in theory, could be released during virolytic oncolysis $[26,27]$. Additionally, melanin is known to bind to a number of potentially toxic drugs and substances, which could result in accumulation of those compounds to toxic levels over time [28]. At this time, we cannot exclude any toxic effects when melanin production is induced by expression of virus-encoded tyrosinase, but we assume that the risk is rather low as melanin is naturally produced in many different organs of many different species. Also, in our studies, we have not detected side effects or adverse events that would indicate major health risks.

To avoid decrease in viral replication due to melanin production, but preserve the advantages given by melanin imaging we constructed an inducible melanogenic rVACV strain. Chen et al. showed previously that the replication rate of $\mathrm{rVACV}$ inversely correlated with the amount of inserted marker genes, which are expressed [29]. Likewise, the viral replication should be enhanced if melanin production is not induced, a finding we observed in our experiments.

In a clinical setting, the melanin production needs to be induced for imaging purposes (or for laser mediated thermotherapy - see below). When sufficient melanin is produced, the dox supplementation would stop and the virus replication may gain speed again. In this study, we did not test in how far the short-term exposure to dox would influence the therapeutic outcome in vivo. However, we could show 
in cell cultures that the duration of doxycycline exposure inversely correlates with the plaque size, and therefore oncolytic potential, of the inducible melanogenic rVACV (Fig. 4). We can therefore assume that short-term dox treatment in patients will not decrease the therapeutic effects.

The synthesized melanin content may be potentially monitored with the vMSOT system employed herein, which can operate as a hand-held probe providing sufficient contrast and nearly isotropic resolution for depths beyond $1 \mathrm{~cm}$ inside tissues [20], in the clinics. Accordingly, indications with surface-near rVACV-colonized tumor lesions can be monitored using optoacoustic imaging systems, whereas colonization in deeper tissues would require MRI.

A further application of the novel rVACV strain is the combination of induced melanin production with laser-induced thermotherapy. This may correct the slower tumors response upon melanin-dependent absorption of near infrared laser light followed by thermal induced cell killing (Stritzker and Kirscher, in preparation).

Additionally, safety is still a major concern in oncolytic viral therapy [30]. Hagen as well as Grigg et al. introduced tet-mediated inducible systems for vaccinia virus based vectors to safely stop viral replication in the host if needed $[31,32]$. If our system is induced, virus replication is not stopped completely but significantly reduced. This melanogenesis associated "metabolic attenuation" can be used as an additional regulatory tool for experiments and in the clinic.

\section{Conclusion}

With the inducible melanogenic rVACV system presented here we were able to image tumor tissues with MRI or MSOT and in addition increased the therapeutic potential of the novel melanogenic oncolytic rVACV based reporter system.

\section{Supplementary Material}

Figures S1-S3, Table S1.

http://www.thno.org/v05p1045s1.pdf

\section{Abbreviations}

AFP: alpha-fetoprotein; CEA: carcinogenic embryonic antigen; CT: computed tomography; dpi: days post induction; dpvi: days post viral infection; dox: doxycycline; hpvi: hours post viral infection; MMP: matrix metalloproteinase; MOI: multiplicity of infection; MRI: magnet resonance imaging; (v)MSOT: (volumetric) multispectral optoacoustic tomography; OD: optical density; PET: positron emission tomography; pfu: plaque forming units; PSA: prostate specific antigen; rVACV: recombinant vaccinia virus strain; SPECT: single-photon emission computer to- mography; (m)Tyr: (murine) tyrosinase; TYRP1: tyrosinase-related protein 1 .

\section{Acknowledgments}

The authors want to thank Johanna Langbein-Laugwitz, Jason Aguilar, Terry Trevino and Sarah Glasl for their excellent technical support. This work was supported by Genelux, graduate stipends from the University of Würzburg (to C.K and L.K.) and ERC Advanced Grant "Next Generation in-vivo imaging platform for post-genome biology and medicine MSOT “ (233161) awarded to V.N.. The publication costs were co-funded by the German Research Foundation (DFG) and the University of Würzburg in the funding program Open Access Publishing.

\section{Competing Interests}

Several of the authors (QZ, JS, AAS) are/were employees and/or shareholders of Genelux Corporation, a company with focus on live vector mediated tumor therapy.

\section{References}

1. Huang TS, Shyu YC, Turner R, et al. Diagnostic performance of alpha-fetoprotein, lens culinaris agglutinin-reactive alpha-fetoprotein, des-gamma carboxyprothrombin, and glypican-3 for the detection of hepatocellular carcinoma: a systematic review and meta-analysis protocol. Syst Rev. 2013; 2 : 37.

2. Horwitz EM, Thames HD, Kuban DA, et al. Definitions of biochemical failure that best predict clinical failure in patients with prostate cancer treated with external beam radiation alone: a multi-institutional pooled analysis. J Urol. 2005; 173: 797-802.

3. Shively JE, Beatty JD. CEA-related antigens: molecular biology and clinical significance. Crit Rev Oncol Hematol. 1985; 2: 355-399.

4. Ntziachristos V. Clinical translation of optical and optoacoustic imaging. Philos Trans A Math Phys Eng Sci. 2011; 369: 4666-4678.

5. Hong H, Sun J, Cai W. Anatomical and molecular imaging of skin cancer. Clin Cosmet Investig Dermatol. 2008; 1: 1-17.

6. Kitai $\mathrm{T}$, Torii $\mathrm{M}$, Sugie $\mathrm{T}$, et al. Photoacoustic mammography: initial clinical results. Breast Cancer. 2012: 1-8.

7. Stritzker J, Kirscher L, Scadeng M, et al. Vaccinia virus-mediated melanin production allows MR and optoacoustic deep tissue imaging and laser-induced thermotherapy of cancer. Proc Natl Acad Sci U S A. 2013; 110: 3316-3320

8. Enochs WS, Petherick P, Bogdanova A, et al. Paramagnetic metal scavenging by melanin: MR imaging. Radiology. 1997; 204: 417-423.

9. Krumholz A, Vanvickle-Chavez SJ, Yao J, et al. Photoacoustic microscopy of tyrosinase reporter gene in vivo. J Biomed Opt. 2011; 16: 080503.

10. Paproski RJ, Forbrich AE, Wachowicz $\mathrm{K}$, et al. Tyrosinase as a dual reporter gene for both photoacoustic and magnetic resonance imaging. Biomed Opt Express. 2011; 2: 771-780

11. Herold MJ, van den Brandt J, Seibler J, et al. Inducible and reversible gene silencing by stable integration of an shRNA-encoding lentivirus in transgenic rats. Proc Natl Acad Sci U S A. 2008; 105: 18507-18512.

12. Chakrabarti S, Sisler JR, Moss B. Compact, synthetic, vaccinia virus early/late promoter for protein expression. Biotechniques. 1997; 23: 1094-1097.

13. Falkner FG, Moss B. Escherichia coli gpt gene provides dominant selection for vaccinia virus open reading frame expression vectors. J Virol. 1988; 62: 1849-1854.

14. Zhang Q, Yu YA, Wang E, et al. Eradication of solid human breast tumors in nude mice with an intravenously injected light-emitting oncolytic vaccinia virus. Cancer Res. 2007; 67: 10038-10046.

15. Stritzker J, Huppertz $S$, Zhang $Q$, et al. Inducible gene-expression in tumors colonized by modified oncolytic vaccinia virus strains. J Virol. 2014

16. Hillen W, Klock G, Kaffenberger I, et al. Purification of the TET repressor and TET operator from the transposon Tn10 and characterization of their interaction. J Biol Chem. 1982; 257: 6605-6613.

17. Ehrig K, Kilinc MO, Chen NG, et al. Growth inhibition of different human colorectal cancer xenografts after a single intravenous injection of oncolytic vaccinia virus GLV-1h68. J Transl Med. 2013; 11: 79.

18. Hess M, Stritzker J, Hartl B, et al. Bacterial glucuronidase as general marker for oncolytic virotherapy or other biological therapies. J Transl Med. 2011; 9: 172. 
19. Hirosaki K, Yamashita T, Wada I, et al. Tyrosinase and tyrosinase-related protein 1 require Rab7 for their intracellular transport. J Invest Dermatol. 2002; 119: $475-480$.

20. Dean-Ben $\mathrm{XL}$, Razansky D. Portable spherical array probe for volumetric real-time optoacoustic imaging at centimeter-scale depths. Opt Express. 2013; 21: 28062-28071.

21. Barza M, Schiefe RT. Antimicrobial spectrum, pharmacology and therapeutic use of antibiotics. Part 1: tetracyclines. Am J Hosp Pharm. 1977; 34: 49-57.

22. Smith K, Leyden JJ. Safety of doxycycline and minocycline: a systematic review. Clin Ther. 2005; 27: 1329-1342.

23. Bahrami F, Morris DL, Pourgholami MH. Tetracyclines: drugs with huge therapeutic potential. Mini Rev Med Chem. 2012; 12: 44-52.

24. Schafer S, Weibel S, Donat $U$, et al. Vaccinia virus-mediated intra-tumoral expression of matrix metalloproteinase 9 enhances oncolysis of PC-3 xenograft tumors. BMC Cancer. 2012; 12: 366.

25. Cunha ES, Kawahara R, Kadowaki MK, et al. Melanogenesis stimulation in B16-F10 melanoma cells induces cell cycle alterations, increased ROS levels and a differential expression of proteins as revealed by proteomic analysis. Exp Cell Res. 2012; 318: 1913-1925.

26. Graham DG, Tiffany SM, Vogel FS. The toxicity of melanin precursors. J Invest Dermatol. 1978; 70: 113-116.

27. Pawelek JM, Lerner AB. 5,6-Dihydroxyindole is a melanin precursor showing potent cytotoxicity. Nature. 1978; 276: 626-628.

28. Larsson BS. Interaction between chemicals and melanin. Pigment Cell Res. 1993; 6: 127-133.

29. Chen NG, Yu YA, Zhang $Q$, et al. Replication efficiency of oncolytic vaccinia virus in cell cultures prognosticates the virulence and antitumor efficacy in mice. J Transl Med. 2011; 9: 164.

30. Chen N, Szalay A. Oncolytic Virotherapy of Cancer. In: Minev BR, editor. Cancer Management in Man: Chemotherapy, Biological Therapy, Hyperthermia and Supporting Measures: Springer Netherlands. 2011: 295-316.

31. Hagen CJ, Titong A, Sarnoski EA, et al. Antibiotic-dependent expression of early transcription factor subunits leads to stringent control of vaccinia virus replication. Virus Res. 2014.

32. Grigg P, Titong A, Jones LA, et al. Safety mechanism assisted by the repressor of tetracycline (SMART) vaccinia virus vectors for vaccines and therapeutics. Proc Natl Acad Sci U S A. 2013; 110: 15407-15412. 